\title{
Philosophiques
}

\section{Le fardeau de la preuve}

\section{Kai Nielsen}

Volume 19, numéro 2, automne 1992

Une nation peut-elle se donner la constitution de son choix?

URI : https://id.erudit.org/iderudit/027198ar

DOI : https://doi.org/10.7202/027198ar

Aller au sommaire du numéro

Éditeur(s)

Société de philosophie du Québec

ISSN

0316-2923 (imprimé)

1492-1391 (numérique)

Découvrir la revue

Citer ce document

Nielsen, K. (1992). Le fardeau de la preuve. Philosophiques, 19(2), 169-190.

https://doi.org/10.7202/027198ar

Ce document est protégé par la loi sur le droit d'auteur. L'utilisation des services d'Érudit (y compris la reproduction) est assujettie à sa politique d'utilisation que vous pouvez consulter en ligne.

https://apropos.erudit.org/fr/usagers/politique-dutilisation/ 


\title{
LE FARDEAU DE LA PREUVE
}

\author{
par \\ Kai Nielsen
}

\section{LE DROIT À I'AUTODÉTERMINATION}

$\mathrm{Si}$, comme je crois que nous devrions le faire, nous justifions les jugements moraux en démontrant qu'ils sont des jugements bien pesés, qui demeurent tels lorsqu'ils sont placés dans un équilibre réfléchi large, alors nous devons accepter - c'est du moins ce que j'avancerai - que des personnes largement majoritaires dans un territoire distinct ont le droit de déterminer leur destinée commune, à condition qu'elles ne violent pas les libertés civiques d'autres personnes, incluant bien sûr les minorités vivant sur le même territoire ${ }^{2}$. Cela signifie que ces personnes ont le droit de déterminer comment elles souhaitent vivre, $y$ compris, très fondamentalement, comment elles souhaitent gouverner leur vie commune. Cela est particulièrement pressant pour un peuple possédant une culture et des traditions distinctes et $y$ attachant une grande valeur. Le fait que de tels peuples aient un tel droit est l'un de nos jugements moraux les plus fondamentaux, du type que John Rawls appelle un de nos

I. Ce texte a été traduit par Geneviẻve Sicotte.

2. Jai articulé mon exposé sur l'appel aux jugements bien pesés dans un équilibre réfléchi large d'après mes ouvrages After the Demise of the Tradition, Boulder, CO, Westview Press, 1991, Pp. 195-248 et Equality and Liberty: A Defense of Radical Egalitarianism, Totowa, NJ. Rowman and Allenheld, I985. pp. 13-44. Voir aussi mes articles « On Needing a Moral Theory: Rationality, Considered Judgments and the Grounding of Morality », Metaphilosophy, 13, avtil 1982, pp. 97-116, et « Sticking with Considered Judgments in Wide Reflective Equilibrium », Philosophia, 13, n’ 3-4, 1985, pp. 316-321. 
jugements bien pesés ${ }^{3}$. De plus, ce n'est pas un jugement bien pesé isolé, mais - c'est du moins ce que j'avancerai - un jugement bien pesé s'intégrant à une structure cohérente d'autres jugements bien pesés, de conceptions morales théoriques et de croyances empiriques qu'auront de manière caractéristique les gens vivant dans des démocraties constitutionnelles modernes. Je ne dis rien sur ce qui pourrait, pour n'importe quelle personne vivant dans n'importe quelles conditions, dans n'importe quel lieu et à n'importe quelle époque, être justifié ou même rationnel. Je soupçonne qu'une telle justification non historique et non contextuelle serait même impossible à concevoir. Quoi qu'il en soit, je parle de justification pour nous, c'est-à-dire pour des gens modernes, adhérant à la démocratie et vivant dans des démocraties constitutionnelles ${ }^{4}$. Je ne crois pas, pour me répéter, que nous puissions parler de manière plausible de ce qui pourrait être justifie pout tout agent rationnel. La justification, qui de toute façon demeure toujours fragile, est beaucoup plus contextuelle et historiquement déterminée que cela $a^{5}$.

3. John Rawls, A Theory of Justice, Cambridge, MA, Harvard University Press, I971. PP. I9-21, 48-51 et 577-587, et John Rawls, « The Independence of Moral Theory », Proceedings and Addresses of the American Philosophical Association XLVII, 1974/75, pp. $7^{-}$Io. Voir aussi Norman Daniels, «Wide Reflec tive Equilibrium and Theory Acceptance in Ethics $»$, Journal of Philosophy, 76 , 1979, et son " Reflective Equilibrium and Archimedean Points ", Canadian Journal of Philosophy, 10, mars 1980. Cette méthodologie a suscité une résistance généralisée dans la communauté philosophique. Certaines des critiques les plus pénétrantes sont venues de sources aussi différentes que Jean Hampton et Joseph Raz. Voir Jean Hampton, « Should Political Philosophy be Done Without Metaphysics? », Ethics, 99, n" 4, I989, Pp. 79I814, et Joseph Raz, "The Claims of Reflective Equilibrium », Inquiry, 25, If 3, 1982, pp. 307-330, et "Facing Diversity: The Case of Epistemic Abstinence 》, Philosophy and Public Affairs, 19, $\Pi^{\prime \prime} 1$, pp. 3-46. J' ai tentè de contrer de tels arguments dans After the Demise of the Tradition, pp. 231-248, et dans « Rawls and the Socratic Ideal », Analyse $\&$ Kritik, $13, \pi^{\circ}$ I, 199I, Pp. $67^{-}$ 93.

4. Voir Rawls sur la justification dans $A$ Theory of Justice, pp. 577-587. Voir aussi sur cette question ses essais plus tardifs: John Rawls, « Kantian Constructivism in Moral Theory », Joumal of Philosophy, 77, 1980, pp. 515552; « The Idea of an Overlapping Consensus », Oxford University Joumal of Legal Studies, 7, 1982, pp. I-25; « The Domain of the Political and Overlapping Consensus ", New York University Law Review, 64, $\Omega^{*}$ 2, 1989, pp. 233-55; et «Justice as Fairness: Political not Metaphysical 》, Philosophy and Public Affairs, 14, n' 2, 1985, pp. 223-251.

5. Dans les essais énumérés plus haut, Rawls dit clairement qu'il n'entre pas dans son ambition de tirer une moralité, même partielle, de la rationalité. I'échec spectaculaire de Alan Gewith et de David Gauthier à démontrer 
Ce que je défends plus haut est ce qui, dans notre jargon politique populaire, a été désigné par le terme vague de droit inhérent à l'autodétermination. Je n'aime pas beaucoup ce type d'expression, car on ignore ce qu'est précisément un droit inhérent, et on ignore aussi ce qu'est précisément un droit. Mais ce qui précède contribue à démythifier partiellement cette expression, et c'est cette conception que je vais défendre et expliciter.

Pour des gens bien informés et aux sentiments démocratiques relativement peu encombrés d'idéologie, les Etats-nations - du moins ceux que nous connaissons - ne constituent guère des objets d'amour, de vénération ou même de grande loyauté. Les Etats n'en demeurent pas moins des maux nécessaires, des instruments essentiels pour assurer la sécurité et la possibilité même d'arriver à quelque chose qui ressemble à une vie confortable ${ }^{6}$. L'anarchie, quand on n'en considère pas les détails pratiques, est un bel idéal, mais hélas, les détails pratiques doivent toujours être considérés. Si nous croyons que les peuples ont un droit à l'autodétermination quand ils sont clairement majoritaires sur un certain territoire et qu'ils ne violent pas les libertés civiques des minorités vivant sur leur territoire et même ailleurs, alors nous devons aussi, pour ce qui est des situations dans lesquelles nous vivons, croire qu'ils ont le droit de former un État-nation ou de protéger un Etat-nation existant de manière à se donner un instrument pour assurer leur autonomie et la conception qu'ils ont d'eux-mêmes en tant que peuple. Mais dans les conditions de rareté modérée qui caractérisent les sociétés développées, la création ou le maintien d'un Etat n'est justifiée que si l'État qui est créé ou qui est maintenu est une démocratie constitutionnelle. Et l'on ne peut pas avoir de démocratie constitutionnelle à moins que les libertés civiques des gens vivant dans cet État ne soient protégées.

L'application de cela au cas du Québec devrait être claire. La société ou la nation qu'est le Québec a le droit de former un Etatnation si elle choisit de le faire. Tout dépend de la décision

un tel lien, malgré le caractère sophistiqué et soigneux de leurs exposés, devtait nous signaler ce que nous aurions dû deviner au dèpart - qu'une telle position est impossible à soutenir. Voir mon ouvrage Why Be Moral?, Buffalo, NY, Prometheus Books, 1989, pp. 245-268, et mon article " Can there be Justified Philosophical Beliefs? », Iyyun, 40, juillet 199I, Pp. 235270.

6. Il y a eu, bien sûr, des sociètès sans État (l'Islande des sagas en était une). Mais dans notre contexte, les Etats sont absolument inévitables. 
réfléchie que prendront les Québécois, et c'est à eux qu'il appartient de déterminer cela démocratiquement. Cela revient dans les circonstances à la détermination par la majorité. Cette décision peut être prise et cet État formé sans que soient violés les droits de qui que ce soit; plus encore, l'État formé devant être une démocratie constitutionnelle, cela doit être fait sans une telle violation. Dans les circonstances, aucun autre système politique ne serait légitime.

Cela implique un droit à la sécession, et même à la sécession unilatérale, pour les peuples placés dans la situation que j'ai décrite. Si ces peuples se perçoivent comme ayant une culture et des traditions distinctes et qu'ils sont largement majoritaires sur un territoire distinct, alors ils peuvent légitimement faire sécession de l'État-nation duquel ils font partie s'ils choisissent de le faire par une décision démocratique, à condition qu'ils ne violent pas les droits d'autres personnes vivant sur le territoire, incluant (bien sûr) les nouvelles minorités qui seraient créees par leur sécession. Il ne s'agit cependant pas ici d'un droit qu'auraient les individus à avoir l'Etat de leur choix, indépendamment du dèsir des autres individus vivant sur le même territoire. Penser cela relèverait d'un individualisme débridé et négligeant complètement la démocratie.

\section{IA CONSCIENCE NATIONAIE}

Supposons que l'on dise, comme l'ont fait les libéraux et les marxistes traditionnels à la suite du rationalisme des Lumières, que l'on ne devrait pas, sauf pour des raisons purement pratiques, se préoccuper de telles identitès locales, de telles identités nationales, le Volksgeist ou Nationalgeist glorifié face au rationalisme des Lumières par Johann Götttfried Herder ${ }^{7}$. Les rationalistes des Lumières croyaient que le fait de se préoccuper de telles questions était un signe d'immaturité, une relique irrationnelle ou même une régression à un passé barbare ou à tout le moins à un ethnocentrisme chauvin et rétrograde enclin à la

7. J. G. Herder, Sümtliche Werke, Berlin, $1877^{-1913 . ~ H e r d e r ~ s o u t e n a i t ~ q u e ~ l e ~}$ Volksgeist ne requerait ni identification ni défense d'un peuple, d'un nationalisme ou d'une religion choisis. Le fait de réaliser, poursuivait-il, que certaines personnes sont notre peuple ne mène pas nécessairement, et en fait ne doit pas mener, à la conclusion que ces personnes sont meilleures ou que leur mode de vie est supérieur. Nous nous identifions avec elles, elles sont notre peuple, mais nous n'avons pas besoin et nous ne devons pas conclure qu'elles forment un peuple superieur. Herder n'est pas Fichte, et encore moins un national-socialiste. 
$x_{\text {xénophobie }}^{8}$. Nous devons, poursuivaient-ils, nous opposer à ces loyautés ethnocentriques. Nous devons être non pas nationalistes, mais internationalistes, et éviter tout attachement local. Cest l'humanité, plutôt que des particularités culturelles enracinées dans la solidarité des groupes, qui doit faire l'objet de notre adhésion réfléchie et qui doit constituer notre conception éthique sous-jacente, une conception profondément humaniste et internationaliste, selon laquelle nous structurons nos entreprises politiques. L'appel au sentiment national - au sentiment d'appartenir à une nation - est toujours une marque d'immaturité et d'ethnocentrisme.

C'est ainsi que raisonnaient à la fois Marx et Comte. Ils considéraient les loyautés nationales ou régionales - les attachements locaux - comme des résistances irrationnelles, reliées aux formes les plus primaires de développement social, et que l'évolution historique à venir rendrait obsolètes. Dans une analyse à ce sujet, G. A. Cohen (nonobstant son marxisme analytique qui devrait lui faire soutenir le contrairel cite une page de Isaiah Berlin, qui à son tour suit dans les grandes lignes Herder, où Berlin affirme qu'il sagit là d'une erreur, d'une erreur qui a empêché un grand nombre de socialistes tout autant que de libéraux de voir la puissance et l'attrait du nationalisme au dix-neuvième et au vingtième siècles ${ }^{9}$. Berlin remarque que le nationalisme est « aujourd'hui un phénomène mondial, probablement le facteur unique le plus puissant dans les Etats nouvellement établis, et dans certains cas chez les populations minoritaires de nations plus anciennes ${ }^{\text {IO }}$. Habituellement, mais pas toujours, ce vif désir « d'indépendance nationale se mêle à une résistance sociale à l'exploitation $»^{\text {II }}$. Il se pourrait bien, poursuit-il, « qu'aucune minorité ayant préservé sa propre tradition culturelle [...] ne puisse tolérer indéfiniment la perspective de demeurer pour toujours une minorité gouvernée par une majorité ayant des conceptions ou des habitudes différentes $\gg{ }^{12}$.

8. Isaiah Berlin, The Crooked Timber of Humanity, New York, Alfred A. Knopf, I99I, p. 248.

9. G. A. Cohen, History, Labour and Freedom, Oxford, Clarendon Press, Ig88, P. $137^{-146 .}$

10. I. Berlin, Op. cit., p. 251. (NDLT: cette citation et les suivantes sont librement traduites).

II. Ibid.

12. Ibid. 
Aucune politique normative voulant sancrer dans le monde réel et éviter de devenir utopique au mauvais sens du terme ne peut se permettre d'ignorer ces considèrations sociologiques et psychologiques. Les peuples souffriront et ne pourront s'épanouir là où ils n'ont pas d'identité sociale stable ${ }^{3}$. Herder a raison de croire que parmi les besoins fondamentaux des êtres humains, il y a, aussi fondamental que le besoin de manger, de se reproduire ou de communiquer, le besoin d'appartenir à un groupe, c'est-à-dire à une communauté quelconque. Mais cela signifie un attachement à une identité locale, et pas seulement à l'humanité en général: ces communautés, ces Gemeinschaften, sont toutes distinctes, ayant leurs formes et leurs structures propres. Les membres de ces communautés sont issus d'un courant de traditions qui modèle profondément leur identité et leur façon d'être, à la fois émotivement et intellectuellement. Berlin formule ainsi la conception de Herder: « Les hommes attachaient (et devraient attacher) une grande valeur aux coutumes, aux activités, aux modes de vie, à l'art et aux idées produits par l'humanité, non pas selon des critères universaux, applicables à tous les hommes et à toutes les sociétés, indépendamment de l'époque et du lieu [...I mais parce que c'étaient les leurs, qu'ils exprimaient leur vie locale, régionale et nationale, et leur parlaient comme ils ne pouvaient parler à aucun autre groupe humain $\gg^{14}$. Si nous voulons trouver un sens aux dimensions morale et politique de notre vie - un sens à notre vie en tant qu'être social - nous devons, poursuit-il, donner un poids moral à nos liens avec des identités particulières enracinées dans une tradition, une langue particulière, une histoire particulière, une région particulière, et le reste. Cela, comme le soutient Herder, ne mène pas nécessairement (et en fait ne devaait pas mener) à la revendication ethnocentrique et agressivement nationaliste selon laquelle notre tradition est supérieure à toutes les autres, ou à une

13. Voir G. A. Cohen, Op. cit., et Kai Nielsen, « Undistorted Discourse, Ethnicity, and the Problem of Self-Definition », dans Winston A van Horne, (dir.), Ethnicity and Language, vol. 6, Milwaukee, WI, University of Wisconsin System, 1987, PP. 16-36, et Kai Nielsen, « Cultural Identity and Self-Definition $\gg$, Human Studies, Io, oct. 1987, pp. 383-390.

14. I. Berlin, Op.cit., p. 245. Voir aussi Isaiah Berlin, Vico and Herder, London, Hogarth Press, 1976, chap. xiii-xxvii, pp. 145-216, et Isaiah Berlin, Against the Current, New York, Viking Press, 1980, pp. 252-286 et 333-355. Pour des arguments solides mais quelque peu différents soutenant que l'appel à l'humanité n'est pas suffisant, voir Richard Rorty, Objectivity, Relativism and Truth, Cambridge, Cambridge University Press, I991, Pp. 21-34 et 175-222. 
revendication voulant que les autres traditions n'aient pas de mérite, en aient un peut-être égal au nôtre, ou même en aient un très grand; cela devrait plutôt nous inciter à reconnaître que c'est parce qu'elle est la nôtre que cette tradition est importante pour nous, et que comme l'a affirmé Émile Durkheim, nous devons, qui que nous soyons, nous avouer à nous-mêmes l'importance de ces attachements locaux si nous ne voulons pas être la proie de l'alienation et du sentiment de n'avoir pas de patrie. Les êtres humains, s'ils veulent échapper à de tels maux, ne peuvent pas être des cosmopolitains déracinés. Ils peuvent, et en fait doivent selon Herder, être des cosmopolitains, mais ils ne peuvent pas être des cosmopolitains déracinés. Il ne suffit pas d'être loyal envers l'humanité; chacun a besoin de sentir qu'il a une patrie, qu'il est membre d'un peuple particulier. Nous ne pouvons pas transcender ou rejeter de telles particularités nous concernant ${ }^{15}$.

Pour parler plus abstraitement, ces particularités culturelles sont nécessaires à la satisfaction des besoins d'identité personnelle et de définition de soi de l'individu. La réalisation de soi et le sentiment de l'identité ne peuvent pas se nourrir uniquement de l'activité créatrice ou de l'identification avec l'humanité. Le développement de pouvoirs humains, les Gattungswesen de Feurbach, qui se ferait indépendamment des définitions de soi particulières et culturellement spécifiques, est probablement impossible, et même s'il est possible, il n'est pas suffisant pour fournir quoi que ce soit qui ressemble à une définition de soi adéquate. Comme le formule bien G. A. Cohen, « un individu n'a pas uniquement besoin de développer et de jouir de ses pouvoirs. Il a besoin de savoir qui il est, et comment son identité le relie à d'autres individus particuliers. Il doit, comme l'a vu Hegel, trouver quelque chose en dehors de lui qu'il n'a pas créé, et à quoi quelque chose en lui correspond, en raison du processus social qui l'a créé, ou en raison d'une redéfinition du moi élaborée par l'expérience ultérieure ${ }^{16}$. Pour dire les choses sommairement, nous sommes perdus si nous ne pouvons pas nous identifier à une partie quelconque d'une réalité sociale objective: une nation, mais pas nécessairement un État, avec ses traditions caractéristiques. On trouve chez les gens le besoin - aussi

15. Berlin fait ressortir avec force et clarté cet aspect de Herder. Voir I. Berlin, Vico and Herder, Op. cit.. Pour une objection soutenant que Berlin a fait Herder plus libéral qu'il ne l'est en réalité, voir Conor Cruise ƠBrien, « Paradise Lost 》, New York Review of Books XXXVIII, 23 avril 199I, Pp. 52-60.

16. Cohen, Op.cit., p. I39. 
profondément ancré que le besoin de développer leurs talents non seulement d'être capables de dire ce qu'ils peuvent faire, mais d'être capables de dire qui ils sont. Cela, on le trouve, on ne le cree pas, et on le trouve dans « l'identification avec les autres dans une culture partagée basée sur la nationalité, la race ou la religion, ou sur une combinaison variable de celles-ci $\gg^{17}$. La nature humaine étant ce qu'elle est, la conscience nationale et la création et le maintien d'une nation sont extrêmement importants pour nous (qui que nous soyons). Dans les conditions contemporaines, cet établissement et ce développement d'une conscience nationale ne peuvent être réalisés qu'à l'aide d'un Etat-nation qui corresponde à cette conscience nationale.

\section{I'ÉtAT-NATION}

Un certain nombre de critiques concernant cette lecture sociale de la définition de soi se présentent immédiatement à l'esprit. Premièrement, on pourrait soutenir que pour qu'une culture soit préservée et que s'épanouisse une conscience nationale, il n'est pas nécessaire qu'un État-nation représente et protège cette conscience nationale. Une nation n'a pas à avoir son propre État-nation qui exprimerait ses intérêts. Tout ce dont nous avons besoin pour notre définition de soi, c'est d'une culture partagée dans un environnement non hostile. Une conscience nationale, un sentiment de former un peuple, comme l'a affirme Herder, qui est tout aussi anti-étatiste que Bakounine, n'a pas besoin d'un État-nation pour s'exprimer.

Il est effectivement vrai qu'un peuple peut avoir un sentiment national sans avoir un État-nation, mais il est aussi vaаi que dans les conditions modernes, cette conscience nationale et l'identité qui lui est associée sont en sûreté uniquement lorsque les peuples ayant ces identités nationales prennent le contrôle de leurs conditions d'existence en obtenant le pouvoir qui accompagne le fait d'avoir leur propre État - un État qui protège et qui promeut activement leurs aspirations nationales. Les Etats multiculturels ou multinationaux n'ont pas très bien fonctionné. Ils n'ont pas réussi à protéger, et encore moins à promouvoir, les identités sociales de leurs diverses cultures. Nous n'avons pas besoin d'invoquer des exemples aussi extrêmes que ceux de la Yougoslavie, de la Hongrie ou de la Roumanie pour voir cela. Même dans des démocraties constitutionnelles aussi établies que la Belgique ou le Canada, les choses ne se sont pas

17. Ibid., p. 140. 
très bien passées. La Finlande et la Suisse semblent être les seules exceptions à cette règle, mais la Suisse fonctionne bien du moins en apparence - grâce à une structure fédérale extrêmement faible, où les cantons ont presque tous les pouvoirs et le contrôle. L'État-nation finlandais est jeune et il subsiste des conflits, bien qu'assez atténués, entre les suédophones et les finnophones qui composent sa population. Ma généralisation semble donc pouvoir être maintenue. Plus encore, quand on examine les grands empires multinationaux abritant plusieurs cultures dans leurs frontières, on voit que fois après fois, ils se sont transformés dans les monstruosités que selon Herder, ils ne pouvaient guère éviter de devenir.

Nous devons donc conclure que les peuples ayant une conscience nationale ont besoin d'un État-nation pour assurer leur identité sociale et pour contrôler leur propre existence. Ils ne pourront avoir cette identité assurée si leur État est contrôlé par des étrangers, et même des étrangers bien intentionnés, ayant des définitions d'eux-mêmes et des aspirations différentes. (Ils ne seraient pas des étrangers s'ils n'étaient pas si différents.) Le désir d'un peuple d'assurer son identité nationale ainsi que le désir d'autonomie qui en découle requièrent qu'un peuple ait son propre Etat-nation.

Mais pourquoi, pourrait-on objecter, se préoccuper de l'identité sociale? Ne voulons-nous pas l'autonomie et le contrôle de nos vies uniquement en tant qu'individus, ou n'est-ce pas, en toute lucidité, ce que nous devrions vouloir? L'identité sociale ou la definition sociale n'ont de valeur qu'en tant qu'elles mènent à cela. Ce qui compte, c'est nous en tant qu'individus, et uniquement en tant qu'individus. L'identité sociale a une valeur purement instrumentale, du reste assez peu fiable. Ces affirmations, bien que familières, sont fausses. Le contrôle sur nos vies que nous voulons est le contrôle sur nos vies non seulement en tant qu'individus, mais aussi en tant que peuple distinct. Et souvenons-nous que nous sommes tous, à des degrés variables de démarcation, membres d'un groupe qui est un peuple distinct ${ }^{18}$.

I8. On soulève parfois une objection de type sociologique post-moderniste que je trouve moi-même troublante. Dans notre "ère post-moderne », diton, où de plus en plus de gens voyagent, vivent dans divers endroits, où des gens ayant des experiences et des antécèdents très différents forment des couples, nous nous retrouvons avec un mélange layant peut-être parfois la vigueur des hybrides) où les gens peuvent ne pas savoir qui ils sont, c'est-à-dire peuvent ne pas avoir une identité sociale clairement delimitée, et ne pas s'en porter plus mal pour autant. En fait, ils peuvent 
En fait, nous ne pourrions guère être des individus, des personnalités distinctes, sans une telle identité sociale. Sans cette identité, nous ne pourrions même pas nous reconnaître en tant qu'êtres humains ayant la capacité d'être autonomes. En fait, comme l'ont montré Steven Lukes et Maurice Mandelbaum dans leurs critiques de l'individualisme méthodologique, un grand nombre des comportements d'un individu ne peuvent pas être identifiés comme actions si l'on ne fait pas référence à une dimension sociale ${ }^{19}$. Aristote reconnaissait que nous sommes des animaux sociaux, et Herder reconnaissait que nous sommes un type d'animal social qui inévitablement a une identité particulière enracinée dans une socialisation particulière. Nous ne sommes pas uniquement, et même peut-être pas du tout, citoyens du monde; nous sommes Finlandais, Québécois, Danois, Catalans, Basques, Français, et ainsi de suite, bien qu'il arrive dans le monde moderne que nos identités soient mêlées. Parfois, notre nationalité a sa patrie dans un Etat qui nous appartient, et parfois elle ne l'a pas, mais ce sont les conditions modernes d'incertitude extrême qui peuvent faire qu'elle n'a pas d'appartenance précise.

Les philosophes, ne pouvant se passer de théorie èthique normative traditionnelle même quand ils reconnaissent notre socialité généralisée, sont aussi susceptibles de poser la question: pourquoi se préoccuper de telles définitions ou identité sociales, sauf pour des raisons pratiques? L'autonomie, la liberté, la réalisation de soi et le plaisir sont toutes des choses

même s'en porter mieux. J'estime que cela demande à être examiné de près, et bien sûr, empiriquement. Je pense qu'il faut dire que ces gens ne sont pas dépourvus d'identité sociale, mais que celle-ci est plus complexe et n'est pas aussi nettement définie que normalement. (Pensons ici en particulier aux enfants de tels couples.) C'est une question essentielle que de savoir s'ils ont besoin de pouvoir cerner précisément cette identité pour échapper a l'aliénation. Je pense que nous devons être agnostiques face à cela. Je suis incapable de cerner précisement ma propre identité sociale, mais pourtant, a moins que je ne me trompe moi-même profondément, je ne souffre pas de cela. Peut-être mon adhésion à la gauche estelle un ersatz pour ces attachements locaux, me protégeant des effets psychologiques fâcheux provenant du fait de ne pas avoir de patrie locale déterminee. Mais cela est loin d'être clair, et évidemment, je suis un cas d'espèce.

19. Steven Lukes, Essays in Social Theory, London, Macmillan Press, I977, Pp. I77186, et Maurice Mandelbaum, « Societal Facts», dans John O'Neill, (dir.), Modes of Individualism and Collectivism, London, Heinemann, 1973. PP. 221234. 
intrinsèquement bonnes, mais l'identité ne l'est pas. Pourquoi voudrions-nous être un certain type de personne? Au lieu de cela, il est plus raisonnable de vouloir être libre, d'être capable de faire ce que nous voulons en tant qu'individus libres de toute interférence, d'être heureux, de satisfaire nos désirs, et le reste. Pourquoi se préoccuper de choses culturelles comme la définition de soi et la compréhension de qui nous sommes?

La réponse vient en deux parties. Premièrement, nous le faisons tout simplement, et si nous tentons de faire abstraction de cela, nous ne serons ni autonomes, ni heureux. Deuxièmement, on pourrait poser exactement la même question au sujet de nos préoccupations pour des choses telles que l'autonomie, la liberté, le bonheur, le plaisir et l'évitement de la souffrance. Pourquoi devrions-nous penser que les quatre premières sont intrinsèquement bonnes et que la dernière est intrinsèquement mauvaise? La réponse, comme l'a montré Henry Sidgwick à propos du plaisir, est que lorsque nous y réfléchissons avec soin et que nous ne souffrons pas de confusion conceptuelle len confondant la bonté intrinsèque avec la bonté instrumentale, par exemplel, nous découvrons tout simplement que nous le faisons. Le plaisir nous apparaît, à la fois initialement et après réflexion, intrinsèquement bon. C'est simplement quelque chose que nous voulons en soi: ce que Georg von Wright considère être la marque de la bonté intrinsèque ${ }^{20}$. On pourrait dire la même chose de la liberté ou de l'autonomie, bien que Sidgwick n'endosserait pas cela. Ce sont des choses dont nous nous préoccupons et que nous voulons pour elles-mêmes. Nous pouvons montrer que nous avons des raisons de les vouloir dans la mesure où elles ont également une utilité pratique, mais nous nous en préoccupons aussi pour elles-mêmes et ne pouvons pas donner de raison pour cela en dehors du fait que quand nous $y$ réfléchissons avec soin et nous préoccupons de la chose, nous découvrons que nous le faisons ${ }^{2 I}$. L'identité de soi sociale a le même statut. Pour revenir aux remarques que je faisais au début de ce texte, il s'agit comme dans les autres cas d'un jugement bien pesé, et ce jugement, tout comme les autres, n'a pas à être

20. Georg Henrik von Wright, The Varieties of Goodness, London, Routledge and Kegan Paul, ig63, p. i18.

21. Max Black, «The Gap Between "Is" and "Should" », dans W. D. Hudson, (dir.), The Is/Ought Question, New York, St Martin's Press, I969, pp. 99-1r3. De plus, même si l'argument soutenu plus haut est erronẻ d'une façon ou d'une autre et que l'identité sociale a seulement une valeur instrumentale, cela n'est certainement pas rien. 
justifié, bien qu'il soit au moins possible d'intégrer les jugements bien pesés dans la structure cohérente d'un équilibre réfléchi large, forme de justification non fondationnelle défendue par Quine, Davidson et Rawls. Si ce recours aux jugements bien pesés soulève trop de questions, on peut, tout en continuant à éviter le fondationalisme, les considèrer comme des préférences bien pesées - des choses que l'on désire pour ellesmêmes - et ces préférences bien pesées peuvent aussi être placées dans un equilibre réfléchi large. Le plaisir, la liberté et l'identité de soi ont tous le même statut ici. Nous n'avons pas plus de raison de traiter l'identité de soi sociale de valeur inacceptable parce que nous ne pouvons pas prouver qu'elle est intrinsèquement bonne que nous n'en avons pour dire que le plaisir n'est pas bon et que la souffrance n'est pas mauvaise parce que nous ne pouvons pas prouver qu'ils sont respectivement bon et mauvais.

\section{LE FARDEAU DE LA PREUVE}

Je veux maintenant aborder un ensemble d'objections tout différent: les objections tournant autour de considerations sur le fardeau de la preuve. Considérant qu'aucun droit n'est absolu, j'ai soutenu en tant que principe fondamental qu'un groupe désireux de former un Etat-nation répondant à ses aspirations a le droit (le droit revendiqué) à l'autonomie nationale, et allant avec lui le droit à la sécession, et si nécessaire à la sécession unilatérale. Quand je parle d'un tel groupe, un groupe formant une nation, et qui pourrait aussi devenir un Etat, je parle d'un groupe vivant sur un territoire distinct (l'habitant depuis longtemps), étant largement majoritaire sur ce territoire, et ayant aussi une culture distincte ${ }^{22}$. Ce groupe a le droit de se séparer de l'Etat duquel il fait partie sil le souhaite et si, ce faisant, il ne viole pas les libertés civiques d'autrui, c'est-à-dire plus généralement s'il le fait en respectant les règles des dèmocraties constitutionnelles et en acceptant les normes du droit international. Là où un tel peuple ainsi réparti dans un territoire a un tel désir et le réalise

22. Il pourrait arriver qu'un peuple, comme ce fut le cas en Islande, s'en aille dans une partie complètement inoccupée du monde et forme un Etat ou une nation qui réponde à ses aspirations culturelles. Mais cela ne s'applique tout simplement pas dans les circonstances contemporaines, où tout le territoire viable est occupé. Aujourd'hui, un peuple dispersé ne pourrait former un Etat-nation qu'à la condition de prendre la place d'un peuple dejà présent sur un territoire, et cela est toujours moralement problèmatique. 
selon les conditions décrites ci-dessus, le droit à la sécession devrait être, moralement parlant, relativement non problématique. Cela reflète la très grande valeur que nous accordons à l'autodétermination, à l'autonomie et au respect de la personne, et le point jusqu'auquel nous croyons que les gens doivent être libres de vivre leur propre vie selon leur désir, à condition qu'ils n'infligent pas certains torts aux autres personnes (en gros les torts decrits par Jonhn Stuart Mill).

Mon exposé adopte une vision plutôt permissive de la sécession comparé à d'autres (par exemple celui d'Allen Buchanan), accordant assez facilement le droit à la sécession (y compris à la sécession unilatérale ${ }^{23}$. Je considère que le fardeau de la preuve revient aux peuples désirant empêcher la sécession; ce sont eux qui doivent ètablir que dans les circonstances particulières qui les occupent, il est légitime de ne pas accorder le droit à la sécession. (Il y aura bien sûr des cas où cela sera légitime.) Certains ont pensé et soutenu (je pense à nouveau à Allen Buchanan) que le fardeau de la preuve devrait être dans l'autre camp. Selon ces derniers, il n'y a pas de droit à la sécession ou à la préservation de la culture, et encore moins à son développement. Le groupe qui fait sécession, a-t-on soutenu, prend une portion du territoire d'un État existant, et il a besoin d'une justification très solide pour le faire. Les biens, et même les biens fondamentaux de la préservation de la culture et de l'autodétermination d'un peuple, ne constituent pas des raisons suffisamment fortes pour justifier une telle prise de territoire. La personne cherchant à légitimer la sécession, poursuit-on, doit montrer que le peuple désirant faire sécession a èté victime d'une profonde injustice, et que la sécession n'entrainera pas une injustice encore plus grande. Dans cette vision, il y a toujours une présomption contre la sécession: elle cause gênéralement de grands torts, et il faut donc avoir une justification extrêmement solide pour s'y engager.

Contrairement à cela, je crois que le droit à la sécession devrait être traité comme le droit au divorce par consentement mutuel. Nous devrions avoir la sécession par consentement mutuel comme nous avons le divorce par consentement mutuel. Dans aucun de ces deux cas, il n'est nécessaire de démontrer une injustice antérieure ou imminente. Si les parties veulent se séparer, dans les deux cas, elles ont le droit de le faire à condition de

$\overline{\text { 23. Alan Buchanan, Secession, Boulder, }}$ CO, Westview Press, I992. 
ne pas infliger certains torts (mais pas tous les torts) à l'autre partie. Si Marie veut se séparer de Michel, elle devrait avoir le droit de le faire à condition qu'il y ait une juste répartition de leurs propriétés communes, que la subsistance et le soin de leurs enfants soient assurés, et ainsi de suite. De la même façon, si le Québec désire se séparer du Canada, il devrait avoir le droit de le faire à condition d'en arriver à un règlement juste concernant la dette et les actifs communs, et le reste. De la même façon que Marie, pour obtenir le divorce, n'a pas à prouver que Michel s'est comporté comme un salaud, le Québec, pour justifier la sécession, ne devrait pas avoir à prouver que de graves injustices ont résulté de l'union du Québec avec le Canada ou, si l'on veut, avec le reste du Canada. (Peut-être y a-t-il eu des préjudices qui ont donné lieu à des injustices et qui demanderaient réparation, mais cela est une autre histoire.) Il n'est pas nécessaire pour justifier le divorce qu'une partie soit coupable. Il suffit que Marie ne désire plus vivre avec Michel. De la même façon, il suffit que le Québec désire quitter le Canada (si c'est ce que le Québec désire) pour justifier la sécession. Le Québec n'a pas à montrer que le Canada lui a causé du tort.

Il me semble que pour des sociétés libérales respectant l'autodétermination, l'autonomie, l'égalité et la démocratie, cette façon de voir à la fois le divorce et la sécession ne devrait pas être problématique. Comme je l'ai remarqué, cela m'a amené à adopter une approche plutôt permissive face à la sécession. Pourquoi certains, tout en demeurant des défenseurs de la société libérale, n'ont-il pas souhaite adopter une telle approche permissive? Pourquoi ces personnes, tout en ayant tendance à adopter une attitude permissive face au divorce, ne le font-elles pas face à la sécession?

Certains diront que s'il n'y a pas eu de cœrcition - une supposition très hypothétique dans la plupart des ententes étatiques - les parties sont liées par un contrat qu'elles ont accepté librement et qu'elles ne peuvent pas rompre sans avoir de très bonnes raisons. Tout comme une personne n'a pas le droit de s'en aller d'un mariage après qu'elle ait cessé de ressentir de l'affection pour son partenaire, un groupe ne peut pas rompre les ententes constitutionnelles et s'en aller d'un État existant quand il n'a plus d'affection politique et ne désire tout simplement plus faire partie de cet ttat. Ces individus et ces groupes n'ont pas le droit, même s'ils en ont le pouvoir, de s'en aller tout simplement. 
On pourrait attaquer cette analogie avec l'argument familier selon lequel ce que signifie le consentement est beaucoup moins clair dans le cas de l'État que dans celui du couple. (Pensons ici aux critiques classiques de Locke.l Quand la majorité d'entre nous a-t-elle signé le contrat social? Mais je laisse cela de côté et je traite les deux cas comme pouvant à bon droit être mis en parallèle. Il est vrai que nous ne pouvons pas simplement nous en aller dans de telles situations. Dans le cas du mariage, si Marie et Michel ont contracté des dettes communes, l'une des parties n'a pas le droit de les ignorer et de laisser l'autre payer les pots cassés. Il doit aussi y avoir une juste division des propriétés et des autres biens que le couple détient ensemble et on doit s'assurer de subvenir aux besoins des enfants qu'ils peuvent avoir. Finalement, et de façon plus controverseee, si l'une des parties, sans en être responsable, a subi des dommages clairs à cause des ententes passées entre les conjoints, tandis que l'autre partie en a bénéficié, la partie favorisèe doit compenser la partie ayant subi des dommages. Ainsi, comme c'est typiquement le cas dans le mariage, si une femme a été économiquement désavantagée parce qu'elle a élevé les enfants tandis que son mari, libre ou plus libre de cette tâche, ètait avantagé par cette entente, une compensation est due à la femme, toutes choses étant égales par ailleurs ${ }^{24}$. Mais cela ne s'appliquera pas à tous les types de torts subis. Si Michel souffre parce qu'il a perdu l'affection de Marie, il n'aura qu'à le supporter, aussi malheureux que cela soit pour lui. On ne peut de toute façon s'assurer de l'affection d'une autre personne par contrat, mais même si cela était possible, ou que la perte d'affection pouvait d'une manière ou d'une autre être compensée, dans une société qui respecte l'autonomie et l'autodétermination, les avantages provenant du fait que les gens puissent vivie leur vie comme ils l'entendent dépassent les effets de tels torts résultant de l'exercice de cette autonomie. Ces torts, bien que reels, ne constituent pas des motifs légitimes pour empêcher un divorce. Une compensation pour l'exploitation subie ou

24. Susan Moller Okin expose brillamment comment, dans le monde moderne, le divorce par consentement mutuel tend a causer des torts aux femmes, et elle montre ce qui doit être fait pour remèdier à cela. Elle ne dit pas que le divorce par consentement mutuel devrait être défendu, mais que ce changement progressif dans la loi sur le divorce, bien que constituant une amélioration par rapport aux pratiques antérieures, comporte encore de très graves problèmes. Voir Susan Moller Okin, Justice, Gender and the Family, New York, Basic Books, I989, pp. I34- I86. 
pour le fait d'avoir été désavantagé, oui, mais pour de tels torts, non.

La même chose se passe pour la sécession. Si, pour prendre le cas qui nous occupe, le Québec désire se séparer du Canada, il ne peut pas légitimement s'en aller sans considérer la dette nationale. Il ne peut pas, et les indépendantistes ne le proposent pas non plus, simplement faire sécession et laisser le reste du Canada assumer la dette nationale dans son entier. De la même façon, le Canada n'a pas le droit d'exiger que le Québec paie pour toutes les propriétés fédérales du territoire qui entreraient en sa possession advenant une telle rupture, alors que les revenus d'impôt destinés à financer ces propriètés ont été perçus équitablement dans toutes les régions du Canada et alors que toutes ces propriétés fédérales ont été localisées de façon raisonnablement équitable dans les diverses régions du Canada. Des deux côtés, il y a des exigences de traitement juste et de division équitable. La nation faisant sécession ne peut donc pas simplement sen aller de son association précédente, particulièrement dans une situation de consentement mutuel (où aucune des parties ne porte une responsabilité plus lourde que l'autre), sans qu'il y ait une division juste de l'actif et du passif de l'Etat duquel elle se sépare. Ici, pour assurer la justice, les deux parties doivent en arriver à un accord de gré à gré, à défaut de quoi elles doivent se soumettre à la decision d'un tribunal international. Ce tribunal ne décide pas si la société a le droit de faire sécession ou noncela n'est de la juridiction d'aucun tribunal - mais se prononce sur ce qui, dans cette situation, constitue une division juste de l'actif et du passif. Encore une fois, de la même façon que Michel souffrira vraisemblablement de la perte de l'affection de Marie, le Canada anglais subira certainement une perte sur le plan culturel si le Québec se sépare. Mais à nouveau, une telle considèration ne peut pas l'emporter sur le droit du Québec à se séparer si c'est ce qu'il choisit de faire. Dans une telle éventualité, c'est simplement une perte que le Canada doit assumer. Le fait de dire que le Québec ne peut pas simplement s'en aller ne comporte aucune présomption contre la sécession, et même contre la sécession unilaterale; cela signifie seulement que pour être légitime, la sécession doit emprunter une certaine voie. Encore ici, la sécession est comparable au divorce. Il est certain que comme dans les cas de divorce, il sera souvent extrêmement difficile de déterminer ce qu'est une division juste, mais cette difficulté n'est pas une impossibilité, et là où, comme dans nos sociétés, l'autonomie et l'égalité sont des jugements bien pesés très 
profondément ancrés, de telles difficultés ne peuvent pas l'emporter sur le droit à la préservation culturelle et sur le droit qu'a un peuple donné de prendre, en tant que peuple, le contrôle de sa propre existence.

Supposons que quelqu'un, s'opposant toujours à ma conception plutôt permissive du droit à la sécession, soutienne, sans tenir compte de mes arguments ci-haut demontrant l'inverse, que la présomption morale doive être contre la sécession étant donne qu'il faut toujours fonctionner avec le présupposé d'un droit des États à l'intégrité territoriale. La propriēté, affirme cet argument, doit être respectée. Nous n'avons pas le droit de diviser des territoires auxquels un État a droit. Seule une injustice extrêmement grave envers le groupe désirant faire sécession, une injustice assez importante pour appeler une rectification, justifierait que l'on divise un territoire de la sorte, une division qui minerait l'intégrité territoriale de l'État. Mais c'est là une autre forme de culte de la propriété. Pourquoi donner un tel poids moral à l'intégrité territoriale des Etats? Les Etats se font et se défont avec les changements de circonstances, incluant des changements dans l'identité sociale. Le Canada, après tout, n'existe que depuis une centaine d'années. De plus, nous avons assisté récemment à la disparition et à l'apparition rapide de nombreux Ėtats. Personne ne sait ou ne peut savoir si les acquisitions initiales étaient justes, et de toute façon, l'Etat n'est qu'un instrument. Ce qui devrait nous préoccuper, ce ne sont pas les droits de propriété et l'intégrité territoriale des États, mais plus fondamentalement, de savoir quels arrangements donneront à un plus grand nombre de personnes un pouvoir presque égal, de manière à donner au maximum de personnes le plus de contrôle possible sur leur vie qui soit compatible avec autant d'autres personnes que possible ayant le même contrôle et les mêmes pouvoirs. L'insistance sur les droits de propriété et sur l'intégrité territoriale des États n'est rien de plus qu'un masque idéologique destiné à protéger le statu quo quel qu'il soit, et cela, de façon générale, empêche les gens de prendre le contrôle de leur propre vie.

Evidemment, la sécession crée de nouvelles minorités, et ces minoritès doivent être traitées équitablement. Le monde étant ce qu'il est, il y aura toujours des minorités. Les francophones sont une minorité au Canada, et les anglophones seraient une minorité dans un Québec souverain. Cela ne constitue une raison ni pour choisir de conserver le statu quo, ni pour le changer. Que les minorités soient vieilles ou récentes, le point crucial est 
que leurs libertés civiques soient protégées. Là où c'est le cas et qu'un groupe culturellement distinct, largement majoritaire et résidant depuis longtemps dans un territoire donné, désire faire sécession d'un Etat duquel il fait partie, ce groupe peut légitimement le faire, même si cela entraîne la création de nouvelles minorités, dans la mesure où les libertés civiques de ces minorités ne sont pas violées. Et il ne suffit pas, pour empêcher la sécession, que le groupe éventuellement minoritaire craigne que ses libertés civiques ne soient pas respectées; il doit réellement exister de bonnes raisons de croire que cela surviendra.

Jusqu'à présent, j'ai considéré les objections morales à ma conception permissive de la légitimité de la sécession. Cependant, il existe aussi des objections d'ordre essentiellement pragmatique et fondées sur la prudence. On affirme qu'il est extrêmement coûteux de passer d'un État existant à un nouvel Etat. Plus encore, cela mène à l'instabilité, mine la sécurité des gens et leur confiance dans l'autorité établie. Leur situation pourrait, relativement à divers aspects très pratiques, devenir pire qu'elle ne l'est.

Je crois qu'ici, nous devons être prudents et y aller cas par cas. Il semble que ces torts (ou des torts comparables) surviendraient dans plusieurs cas, mais pas dans tous. Dans l'ancienne Union Soviétique et dans l'ancienne Yougoslavie, ces torts ou plusieurs d'entre eux ont effectivement lieu. Par contre, cela n'a pas été le cas quand l'Islande et la Norvège se sont séparées du Danemark. Là où il y a une longue histoire d'injustice, de méfiance et de haine mutuelles, de chauvinisme, d'intolérance religieuse et raciale et de xénophobie, on peut s'attendre à ce que les choses tournent mal, comme dans les cas de la Yougoslavie et de la Roumanie. Dans ces circonstances, il y aura une grande insécurité, et beaucoup de gens subiront des torts (pour dire le moins); cependant, il y avait déjà dans la situation présécessionnaire beaucoup de cela, ainsi que d'autres choses très graves comme par exemple la domination totalitaire ou autoritaire. Il est très difficile de déterminer quelle est la situation qui générera le plus de torts. Nous devons y aller cas par cas et être très prudents dans nos généralisations.

Là où ces choses manifestement mauvaises ne surviennent pas ou surviennent seulement sous une forme très attènuée, il n'y a pas de raison de croire que la sécession sera si coûteuse. La Scandinavie est un exemple de cela; de même, si les Gallois et les Ecossais desirent suivre leur propre chemin en dehors du 
Royaume-Uni, il n'y a pas de raison valable pour supposer que le coût en sera élevé. On peut plutôt penser que ce coût ne sera pas élevé puisque la situation dans ces cas en serait une où les croyances des gens - essentiellement la l'intensité de leur adhésion à la démocratie, leur respect des droits individuels et leur capacité d'être raisonnable - constitueraient des freins cruciau $^{25}$. Si le Québec devait prendre la voie de l'indépendance, il n'est pas déraisonnable d'espérer, et même de s'attendre, à ce que les deux parties se montrent relativement raisonnables. Evidemment, il y aurait avant la sécession beaucoup d'hypocrisie et des magouilles idéologiques (une chose à laquelle nous assistons presque tous les jours), mais après qu'elle serait devenu un fait accompli, les choses prendraient un cours plus raisonnable. Les convictions relatives à la democratie et au respect des droits sont solidement ancrées dans la culture des deux nations. Et même si l'on peut raisonnablement penser que la situation serait quelque peu enflammée, le Québec garderait un couloir ouvert entre les Maritimes et l'Ontario, et le Canada ne tenterait pas de soutirer le maximum au Québec et protégerait les droits des francophones hors-Québec. Les deux parties verraient la nécessité de la coopération future, et ne formuleraient pas des exigences déraisonnables et d'une injustice flagrante qui mineraient la possibilité d'une coopération future tout en leur donnant mauvaise réputation sur le plan international.

Peut-être suis-je trop optimiste dans ma vision des choses, mais l'histoire nous fournit des précédents parmi des pays relativement aisés ayant une longue tradition de démocratie constitutionnelle et un certain niveau d'éducation. Lorsque le Danemark et l'Islande se sont séparés, il y a eu de l'amertume des deux côtés, mais les deux pays y ont fait face de façon raisonnable et sont maintenant de bons voisins ${ }^{26}$. Le Canada et le Québec

25. La capacité d'être raisonnable, considérée comme distincte de la rationalité, est directement une qualité morale. Jai utilisé cette distinction, tout comme Brian Barry et John Rawls. Voir mon article « Distrusting Reason », Ethics, 87, oct. 1976, pp. 49-60. Pour les textes classiques à ce sujet, voir W. M. Sibley, « The Rational Versus the Reasonable », Philosophical Review, 62, 1953, Pp. 554-560, et R. S. Peters, « The Development of Reason », dans S. I. Benn et G. W. Mortimore, (dir.), Rationality in the Social Sciences, London, Routledge and Kegan Paul, 1976, pp. 299-331.

26. Les choses ne se passèrent pas toujours de façon aussi civilisée en Scandinavie. En 1520, l'armee du roi Christian II du Danemark massacra un grand nombre d'habitants de Stockholm. 
ne sont pas - ou à tout le moins ne devraient pas être différents à ce sujet.

\section{UNE CRISE CONSTITUTIONNELLE?}

Pour terminer, on pourrait me poser la question suivante: «Quel rapport y a-t-il entre la thèse que vous avez soutenue et la crise constitutionnelle? Pourquoi parler de sécession et d'indépendance, quand la question est de savoir quels nouvelles ententes constitutionnelles doivent, s'il y a lieu, être passées entre le Québec et le reste du Canada? » Si j'ai insisté sur les points développés plus haut, c'est que je crois que le fait même de conceptualiser la question en tant que crise constitutionnelle, particulièrement du point de vue du Québec, est une façon trompeuse et erronée de caractériser la situation. Certains considèrent déjà acquis le fait que le Québec se séparera et deviendra indépendant. Si c'est le cas, il n'y a pas de crise constitutionnelle pour le Québec. Les nouveaux Ėtats-nations ne sont manifestement pas liès par de vieilles constitutions, qu'il s'agisse de leur propre constitution en tant que province ou colonie avant qu'ils ne deviennent des États indépendants, ou de la constitution d'un Etat duquel ils veulent se séparer. Là où la situation n'en est pas une de révolution, ces nouveaux Etats ne doivent pas, en recherchant et en maintenant leur indépendance, violer les normes du droit international, mais c'est là une autre question. Leur tâche est d'élaborer une nouvelle constitution qui leur soit propre sans se préoccuper des questions constitutionnelles de l'État-nation duquel ils veulent se séparer, bien qu'ils doivent fonctionner à l'intérieur des paramètres de ce qui est permis aux démocraties constitutionnelles.

Les mêmes considérations demeurent valides si le fond du débat au Québec est de savoir sil faut ou non devenir un Étatnation indépendant, et si cela n'est pas une question résolue d'avance mais un choix véritable. C'est uniquement quand cette question sera résolue que la question constitutionnelle se portera au premier plan, et encore uniquement si elle est résolue d'une certaine façon. Si le Québec opte pour l'indépendance, la question constitutionnelle ne se posera pas: seuls seront à l'ordre du jour des problèmes de marchandage et de négociation juste entre le Québec et le Canada. (Je prends pour acquis que le Canada, contrairement au Nord durant la Guerre de Sécession américaine, ne tentera pas de maintenir l'union par la force.)

Si le Québec rejette l'indépendance, et tente de demeurer au sein du Canada mais avec un statut différent, alors la question 
constitutionnelle se pose à nouveau. Mais cela demande que soit résolue la première question, c'est-à-dire la question de l'indépendance.

Ce qui précède, à ce qu'il me semble, ne demande que les réserves suivantes. Certains Ouébécois veulent laisser l'option de l'indépendance en suspens jusqu'à ce qu'ils puissent voir les nouveaux arrangements constitutionnels que les autres provinces et le gouvernement fédéral seraient prêts à accepter, et qu'ils puissent déterminer si ces arrangements seraient plus à la convenance d'un Québec recherchant une plus grande autonomie que cela n'est possible dans les arrangements actuels. Si c'est le cas, alors les questions constitutionnelles sont cruciales. Jai supposé, peut-être à tort, que ce n'est pas le cas, et que la question est essentiellement de savoir si le Québec doit chercher à devenir un État souverain ou non. Cest évidemment une intuition politique de ma part. Si ma supposition, basée sur cette intuition, est correcte, c'est donc une erreur que de caractériser la crise actuelle comme une crise constitutionnelle. J'ai raisonné en conformité avec cette supposition dans les dernières parties de cet essai.

Il ne m'appartient pas, moi qui suis nouveau venu au Québec et de surcroît intellectuel déraciné, de même suggérer aux Québécois ce qu'ils devraient faire. J'ai uniquement voulu soutenir que si les Québécois prennent le chemin de l'indépendance, ils peuvent le faire sans violer les droits de qui que ce soit, et sans rien faire d'autre qui soit injuste ou de quelqu'autre façon moralement fâcheux ou imprudent ${ }^{27}$.

\section{Departement de philosophie}

Université de Calgary

27. Une version abrégée de ce texte a été présentee au colloque « Une nation peut-elle se donner la constitution de son choix? », tenu le i2 mai 1992 au congrès de l'Association canadienne-française pour l'avancement des sciences (ACFAS) à l'Université de Montréal. Dans cette note, je commenterai brièvement certaines affirmations de l'un des participants à ce colloque, Allen Buchanan. Buchanan a montré, correctement à mon avis, que la souveraineté n'est qu'une des formes que peut prendre l'autodètermination nationale. L'autodétermination peut prendre bien des formes en dehors de la souveraineté, et évidemment, l'autodétermination nationale n'entraîne pas necessairement la souveraineté; mon argument est plutôt que sans la souveraineté, l'autodétermination nationale n'est pas assurée, 
du moins pas dans les conditions modernes. Buchanan rejette tout principe d'un droit genéral à l'autodétermination, faisant valoir qu'il $y$ a plus de nations voulant exercer leur droit à l'autodétermination qu'il n'y a de territoire sur lequel elles peuvent réaliser ce droit La satisfaction de ce droit pour certains menerait à sa privation pour les autres. Mais le droit à l'autodètermination, comme tout autre principe fondamental, comporte des clauses ceteris paribus, et doit selon moi pouvoir cadrer avec d'autres principes. Il ne constitue pas un principe supréme unique. Il arrive qu'il ne puisse pas être appliqué, dans des situations où son application amoindrirait des principes encore plus moralement pressants. Mais cela à mon avis est vrai de tout principe moral, et n'invalide pas ces principes pour autant Buchanan affirme aussi que l'application de ce principe pourrait mener à une fragmentation illimiteee. Cela est possible, sans doute, mais cela est-il vraisemblable? On peut mème soutenir qu'il est préférable d'avoir davantage de petits Etats correspondant de plus près au sentiment national de différents peuples. A une certaine dimension - mais il n'y a pas de raison de penser que nous pouvons déterminer quand cela survient précisément - la région serait trop petite pour constituer un Etat viable dans les conditions modernes. Il semble peu plausible que l'île du Prince-Edouard ou que les iles Féroè puissent former des Etats-nations viables. Mais à un certain moment, la prudence et la raison se manifesteraient, et les gens ne chercheraient pas à former un Etat-nation, ou ne seraient pas justifiés s'ils le faisaient. Ils pourraient avoir le droit de le faire (cela pourrait être permis moralement), mais ne pas être justifiès de le faire. Ce que nous avons le droit de faire peut ne pas toujours être la bonne chose à faire. C'est parfois une erreur que d'exercer ce qui constitue tout simplement nos droits. On ne doit pas confondre « avoir le droit de faire $x$ » avec « $x$ est la bonne chose à faire ». 